\title{
Bioinformatics analysis of the target gene of fibroblast growth factor receptor 3 in bladder cancer and associated molecular mechanisms
}

\author{
XING AI $^{1 *}$, ZHUO-MIN JIA ${ }^{1,2^{*}}$, JUAN WANG $^{3}$, GUI-PING DI ${ }^{1}$, XU ZHANG $^{2}$, \\ FENGLING SUN ${ }^{1}$, TONG ZANG $^{1}$ and XIUMEI LIAO ${ }^{1}$ \\ ${ }^{1}$ Department of Urology, Military General Hospital of Beijing PLA, Beijing 100700; \\ ${ }^{2}$ Department of Urology, Chinese PLA General Hospital, Beijing 100853; \\ ${ }^{3}$ Department of Medicine, Military General Hospital of Beijing PLA, Beijing 100700, P.R. China
}

Received August 4, 2014; Accepted April 24, 2015

DOI: $10.3892 / \mathrm{ol} .2015 .3231$

\begin{abstract}
The aim of the present study was to elucidate the molecular mechanisms of fibroblast growth factor receptor 3 (FGFR3) activation via overexpression or mutation of the FGFR3 target gene in bladder cancer (BC). The transcription profile data GSE41035, which included 18 BC samples, containing 3 independent FGFR3 short hairpin (sh)RNA, and 6 control samples, containing enhanced green fluorescent protein (EGFP) shRNA, were obtained from the National Center of Biotechnology Information Gene Expression Omnibus database. The Limma package with multiple testing correction was used to identify differentially expressed genes (DEGs) between FGFR3 knockdown and control samples. Gene ontology (GO) and pathway enrichment analysis were conducted in order to investigate the DEGs at the functional level. In addition, differential co-expression analysis was employed to construct a gene co-expression network. A total of 196 DEGs were acquired, of which 101 were downregulated and 95 were upregulated. In addition, a gene signature was identified linking FGFR3 signaling with de novo sterol biosynthesis and metabolism using GO and pathway enrichment analysis. Furthermore, the present study demonstrated that the genes NME2,
\end{abstract}

Correspondence to: Dr Zhuo-Min Jia, Department of Urology, Military General Hospital of Beijing PLA, 5 Nanmeng Cang Road, Dongcheng, Beijing 100700, P.R. China

E-mail: zhuominjiazhj@163.com

Dr Xu Zhang, Department of Urology, Chinese PLA General Hospital, 28 Fuxing Road, Haidian, Beijing 100853, P.R. China

E-mail: xuzhangxx@163.com

${ }^{*}$ Contributed equally

Key words: bladder cancer, differentially expressed genes, gene ontology and pathway enrichment analysis, protein-protein interaction network, differentially co-expressed genes
CCNB1 and H2AFZ were significantly associated with BC, as determined by the protein-protein interaction network of DEGs and co-expressed genes. In conclusion, the present study revealed the involvement of FGFR 3 in the regulation of sterol biosynthesis and metabolism in the maintenance of $\mathrm{BC}$; in addition, the present study provided a novel insight into the molecular mechanisms of FGFR3 in BC. These results may therefore contribute to the theoretical guidance into the detection and therapy of $\mathrm{BC}$.

\section{Introduction}

Bladder cancer (BC) is estimated to be among the top five most common types of cancer in western countries and ranks number 13 in terms of cancer-associated mortality worldwide $(1,2)$. Histologically, BC may be classified based on the depth of invasion: pTa, papillary; pT1, lamina propria invasion; pT2, muscle invasive; pT3, invasion to peri-vesical fat; and pT4, locally advanced $(3,4)$. BC results from long-term exposure to contaminants or other environmental factors involving gene mutations and progressive cellular damage. Parkin et al (5) demonstrated that the incidence of BC is almost four times higher in men than in women and inducing factors include tobacco smoke, prolonged exposure to chemical substances and race $(6,7)$.

Genetic mutations in gene expression may lead to the malignant transformation of bladder cells. High-throughput DNA microarray analyses have identified multiple DNA mutations and alterations in the genesis of $\mathrm{BC}$; these include genes encoding for B cell lymphoma-2, p53, H-Ras and fibroblast growth factor receptor 3 (FGFR3) $(8,9)$. FGFR3 is a tyrosine kinase receptor that regulates fundamental developmental pathways and triggers a range of cellular processes, including proliferation, differentiation, migration and apoptosis (10). The FGFR3 gene is located on chromosome region 4p16.3 (11) and it is made up of 9 exons and 18 introns (12). The structure of FGFR3 is composed of an extracellular domain consisting of two or three immunoglobulin-like domains, a hydrophobic transmembrane domain and an intracellular tyrosine kinase domain (13). Upon ligand binding, FGFR3 forms dimers 
and activates the intracellular kinase domain, resulting in autophosphorylation of this domain. The phosphorylated residues are the binding targets of the adaptor proteins and their binding results in the activation of several signal transduction pathways, including the Ras mitogen-activated protein kinase (MAPK) signaling pathway and phosphoinositide 3-kinase (PI3K) Akt mammalian target of rapamycin (mTOR) pathway (14).

There are two mechanisms to explain the abnormal activation of FGFR3: Overexpression or activating mutations. FGFR3 mutations have been identified in multiple dwarfisms (15), such as hypochondroplasia, and in multiple types of cancer, including prostate cancer (16), cervical cancer (17) and BC. FGFR3 mutations were reported in BC for the first time by Cappellen et al (18). There is evidence to suggest that codons 248, 249 and 375 are the major mutation hot spots in BC (19); in addition, activating mutations of FGFR3 have been revealed primarily in pTa (60-70\%) and in pT1-4 (16-20\%) (19). Overexpression of FGFR3 has been frequently identified in BC; furthermore, Jebar et al (20) demonstrated that the expression of FGFR3 was higher in low stage BC. Du et al (21) identified a gene pathway linking FGFR3 with sterol and lipid metabolism through transcriptional profiling of BC cells subjected to short hairpin (sh)RNA knockdown of FGFR3 (21). FGFR3 has been demonstrated to be a promising therapeutic target for BC $(22,23)$. However, the molecular mechanisms of FGFR3 activation, via overexpression or activating mutation, in $\mathrm{BC}$ remain to be elucidated.

The present study aimed to analyze microarray data in order to investigate the changes in gene expression profiles that occur following loss of FGFR3; in addition, the current study aimed to explore the target genes and molecular mechanisms of FGFR3 The genes that were differentially expressed in FGFR3-deleted cell lines as compared with the control cell lines were considered to be potential transcriptional targets of over-expressed FGFR3 in bladder cancer. Furthermore, a protein-protein interaction (PPI) network was constructed and the disturbed biological pathways were identified following FGFR3 knockdown in order to explore the pathogenesis and occurrence of BC associated with FGFR3.

\section{Materials and methods}

Messenger RNA expression profile data of BC. The transcription profile dataset of $\mathrm{BC}$ was obtained from National Center of Biotechnology Information Gene Expression Omnibus database (http://www.ncbi.nlm.nih.gov/geo/). The accession number was GSE41035 and the dataset consisted of a total of 24 mRNA samples, including 18 experimental samples collected from RT112 cell lines, with FGFR3 shRNA 2-4, FGFR3 shRNA 4-1 or FGFR3 shRNA 6-16, as well as 6 control enhanced green fluorescent protein (EGFP) shRNA samples. The platform used was GPL570 Affymetrix Human Genome U133 Plus 2.0 array (Affymetrix, Inc., Santa Clara, CA, USA). The original CEL files and the annotations file were downloaded based on this platform.

Identification of differentially expressed genes (DEGs). Probe-level data in the CEL files were first converted into expression measures. For each sample, the expression values of all probes for a given gene were reduced to a single value by taking the average expression value. Subsequently, missing data was imputed and quartile data normalization was performed by robust multichip averaging using Affy package in $\mathrm{R}$ software (version 3.1; http://www.bioconductor. org/packages/release/bioc/html/affy.html) (24). The Limma package version 3.24.2 (http://www.bioconductor.org/packages/release/bioc/html/limma.html) (25) in R language with multiple testing correction was then used according to the Benjamini \& Hochberg method (26) in order to identify DEGs between BC samples and normal controls. $\mathrm{P}<0.05$ and $\log$ (fold change; FC) $\mid>1$ were defined as the thresholds.

Gene ontology $(G O)$ enrichment analysis. In order to investigate DEGs at the molecular and functional level, the online biological tool, Database for Annotation, Visualization and Integrated Discovery (DAVID) version 6.7 (http://david. abcc.Ncifcrf.gov/), was used for GO term enrichment and genes were clustered according to GO. GO is a collection of controlled vocabularies, which include molecular function, cellular component and biological process, to describe the biology of a gene product in any organism. $\mathrm{P}<0.05$ was selected as the cut-off criterion during the analysis.

Pathway enrichment analysis. The theoretical principle for enrichment analysis is that associated functional genes are more likely to be selected in the abnormal biological process by the high-through screening technologies (27). Based on the selected genes, researchers are able to correctly identify the biological processes involved. In order to identify the enriched pathways of DEGs, DAVID was used with $\mathrm{P}<0.05$ as the threshold. The pathways used as DAVID input for cluster analysis were from Kyoto Encyclopedia of Genes and Genomes (KEGG; http://www.genome.jp/kegg/) and BIOCARTA (http://www.biocarta.com/).

PPI network construction. PPIs are crucial for all biological processes. In the present study, the PPI network was constructed based on the Protein Interaction Network Analysis platform (PINA2) database (http://cbg.garvan.unsw.edu. au/pina/). PINA2 (28) is a database containing known and predicted associations of protein interaction. The interactions include direct (physical) and indirect (functional) associations. Of note, the protein names in the Universal Protein Resource database (http://www.ebi.ac.uk/uniprot/remotingAPI/), which correspond to the DEGs, were submitted to construct the PPI network. Here Cytoscape software (version 3.2.1; http:// cytoscape.org/) (29) was used to visualize the PPI network to further observe the associations between genes.

Analysis of co-expressed genes. DEGs only explain a limited number of mechanisms of FGFR3-shRNA in BC. However, analysis of differential co-expression genes may reveal two or several similar genes with similar expression patterns across a set of samples. Co-expression genes were hypothesized to have a functional association, such as physical interaction between the encoded proteins $(30,31)$. In order to further explore the pathogenesis of BC in the present study, the differential coexpression enrichment (DCe) function in DGCL package (32) version 2.1.2 (http://cran.r-project.org/ 
Table I. Clustering of differentially expressed genes based on biological process.

\begin{tabular}{llrr}
\hline GO ID & \multicolumn{1}{c}{ GO name } & Gene number & P-value \\
\hline GO:0016126 & Sterol biosynthetic process & 8 & $8.87 \times 10^{-8}$ \\
GO:0016125 & Sterol metabolic process & 11 & $1.58 \times 10^{-7}$ \\
GO:0008202 & Steroid metabolic process & 14 & $3.52 \times 10^{-7}$ \\
GO:0008203 & Cholesterol metabolic process & 10 & $7.47 \times 10^{-7}$ \\
GO:0055114 & Oxidation reduction & 22 & $6.80 \times 10^{-6}$ \\
GO:0006695 & Cholesterol biosynthetic process & 6 & $8.27 \times 10^{-6}$ \\
GO:0044421 & Extracellular region part & 27 & $3.78 \times 10^{-5}$ \\
GO:0006694 & Steroid biosynthetic process & 8 & $4.11 \times 10^{-5}$ \\
GO:0042802 & Identical protein binding & 5 & $4.16 \times 10^{-5}$ \\
GO:0008299 & Isoprenoid biosynthetic process & $5.97 \times 10^{-5}$ \\
\end{tabular}

${ }^{a}$ Number of differentially expressed genes enriched in this GO term. GO, gene ontology.

Table II. Biological pathways in bladder cancer cells.

\begin{tabular}{llcl}
\hline Category & \multicolumn{1}{c}{ Pathway name } & P-value & \multicolumn{1}{c}{ Gene name } \\
\hline KEGG & Steroid biosynthesis & $4.80 \times 10^{-5}$ & \multicolumn{1}{c}{ CYP51A1, SQLE, DHCR7, FDFT1, } \\
& & & SC4MOL \\
KEGG & Terpenoid backbone biosynthesis & $7.77 \times 10^{-4}$ & HMGCR, FDPS, IDI1, ACAT2 \\
BIOCARTA & Fibrinolysis pathway & 0.009393 & SERPINB2, PLAU, F2R \\
KEGG & Arachidonic acid metabolism & 0.03285 & PLA2G4A, PLA2G10, GPX3, CYP4F3 \\
KEGG & Complement and coagulation cascades & 0.045395 & F5, CFD, PLAU, F2R \\
BIOCARTA & Nuclear receptors in lipid metabolism & 0.047437 & CYP24A1, ABCG1, CYP4B1 \\
& and toxicity & & \\
\hline
\end{tabular}

KEGG, Kyoto Encyclopedia of Genes and Genomes.

web/packages/DCGL/index.html) in R language was used and the parameters in the function were set to default values. $\mathrm{P}<0.05$ and the maximum absolute correlation coefficient $>1.5$ were set as thresholds.

\section{Results}

Identification of DEGs. The Limma package was used to analyze the transcription profile data between the experimental and control samples. $\mathrm{P}<0.05$ and $\mid \log \mathrm{FCl}>1$ were used as the significant thresholds for DEGs. Based on these criteria, a total of 196 DEGs were identified, among which 101 were downregulated and 95 were upregulated.

GO analysis. Functional classification was performed using the online biological tool DAVID, with a threshold of $\mathrm{P}<0.05$. Table I demonstrates the top ten significantly enriched GO terms when these DEGs were classified according to biological process. This analysis revealed that the most enriched functions detected in FGFR3 knockdown samples compared with control samples were the biosynthesis of sterol $\left(\mathrm{P}=8.87 \times 10^{-8}\right)$ and metabolism of sterol $\left(\mathrm{P}=1.58 \times 10^{-7}\right)$ or steroid $\left(\mathrm{P}=3.52 \times 10^{-7}\right)$. In addition, oxidation reduction, extracellular region part and identical protein process were also demonstrated to be enriched.
Analysis of the biological pathways of DEGs. The gene transcription profile was significantly altered in experimental samples compared with control samples. These DEGs were selected for KEGG and BIOCATRA pathway enrichment analysis. As shown in Table II, according to the threshold of $\mathrm{P}<0.05$, six biological pathways were significantly enriched. Consistent with the results of the GO enrichment analysis, these pathways were primarily associated with biosynthesis and metabolism, including steroid biosynthesis $\left(\mathrm{P}=4.80 \times 10^{-5}\right)$ and arachidonic acid metabolism $(\mathrm{P}=0.03285)$.

PPI network construction. DEGs were mapped to a PINA2 database and a PPI network was constructed. The PPI network identified 1,865 genes, with 2,482 interactions between them (Fig. 1). Genes that had more interactions with other genes may have a critical role in $\mathrm{BC}$. Table III demonstrates the DEGs with the ten highest degrees of gene interactions identified in these samples. The gene SETX was reported to have the highest degree (degree, 159), which indicated that it may have an important role in FGFR3-regulated BC.

Analysis of co-expressed genes. Co-expressed genes were identified using DCe function in R language; a gene co-expression network was then constructed and visualized 


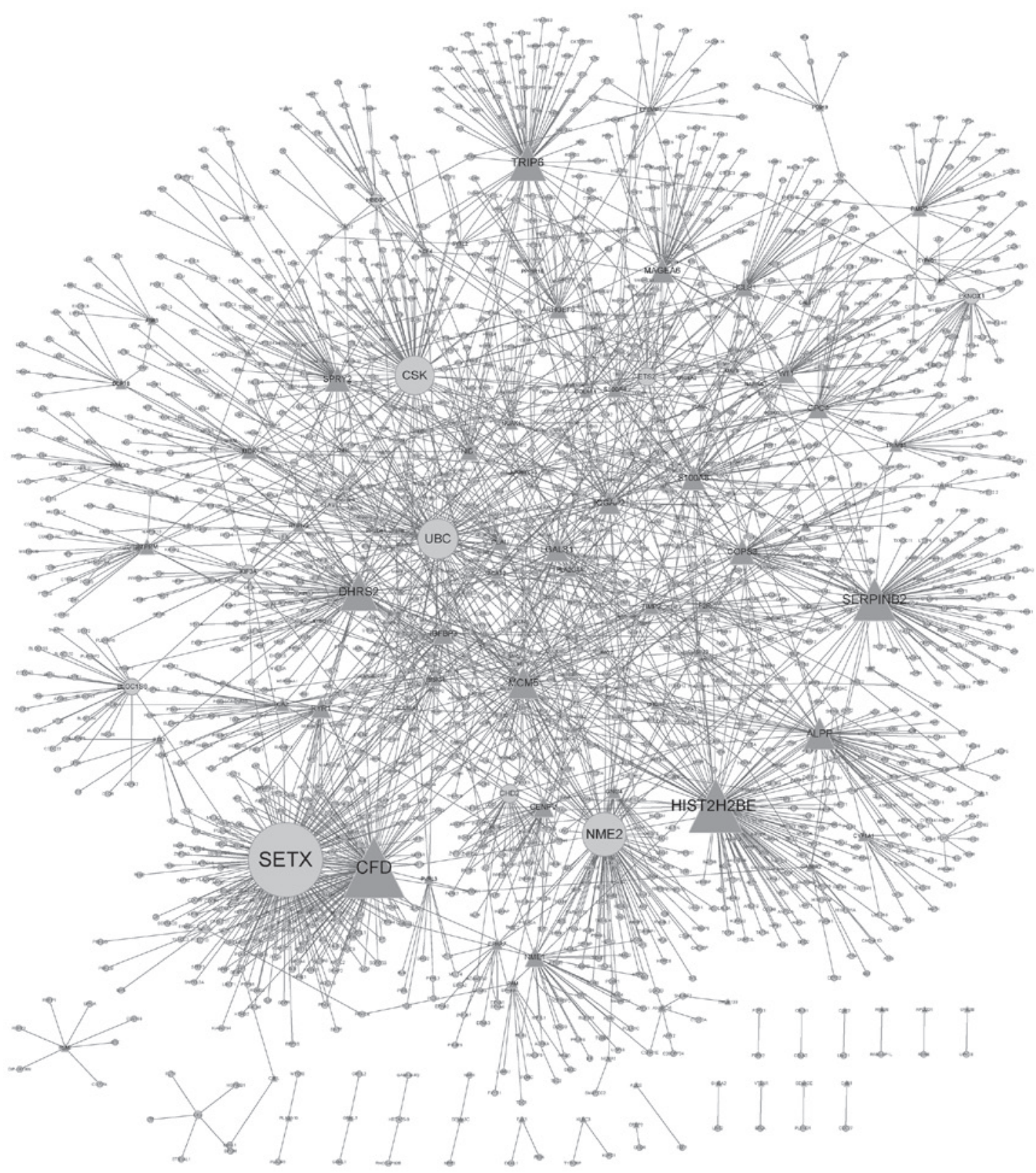

Figure 1. Protein-protein interaction network for products of DEGs. A total of 1,865 gene nodes and 2,482 interaction associations were identified. Triangular nodes, 146 DEGs; circular nodes, 1,719 non-DEGs. DEGs, differentially expressed genes. For each node, the degree represents the number of interactions with other nodes and node size is proportional to the degree of each node.

Table III. Ten highest degrees of interaction and the corresponding differentially-expressed genes.

\begin{tabular}{lc}
\hline Gene name & Degree \\
\hline SETX & 159 \\
CFD & 127 \\
HIST2H2BE & 110 \\
NME2 & 88 \\
SERPINB2 & 85 \\
UBC & 80 \\
CSK & 77 \\
DHRS2 & 75 \\
TRIP6 & 72 \\
MCM5 & 60 \\
\hline
\end{tabular}

Table IV. Ten highest degrees of interaction and the corresponding differentially co-expressed genes.

\begin{tabular}{lc} 
Gene name & Degree \\
\hline CCNB1 & 39 \\
MCM3 & 26 \\
TMEM97 & 25 \\
MCM5 & 25 \\
H2AFZ & 25 \\
PPIL1 & 25 \\
UBE2T & 22 \\
MCM2 & 21 \\
CTSF & 19 \\
ZIC2 & 19
\end{tabular}




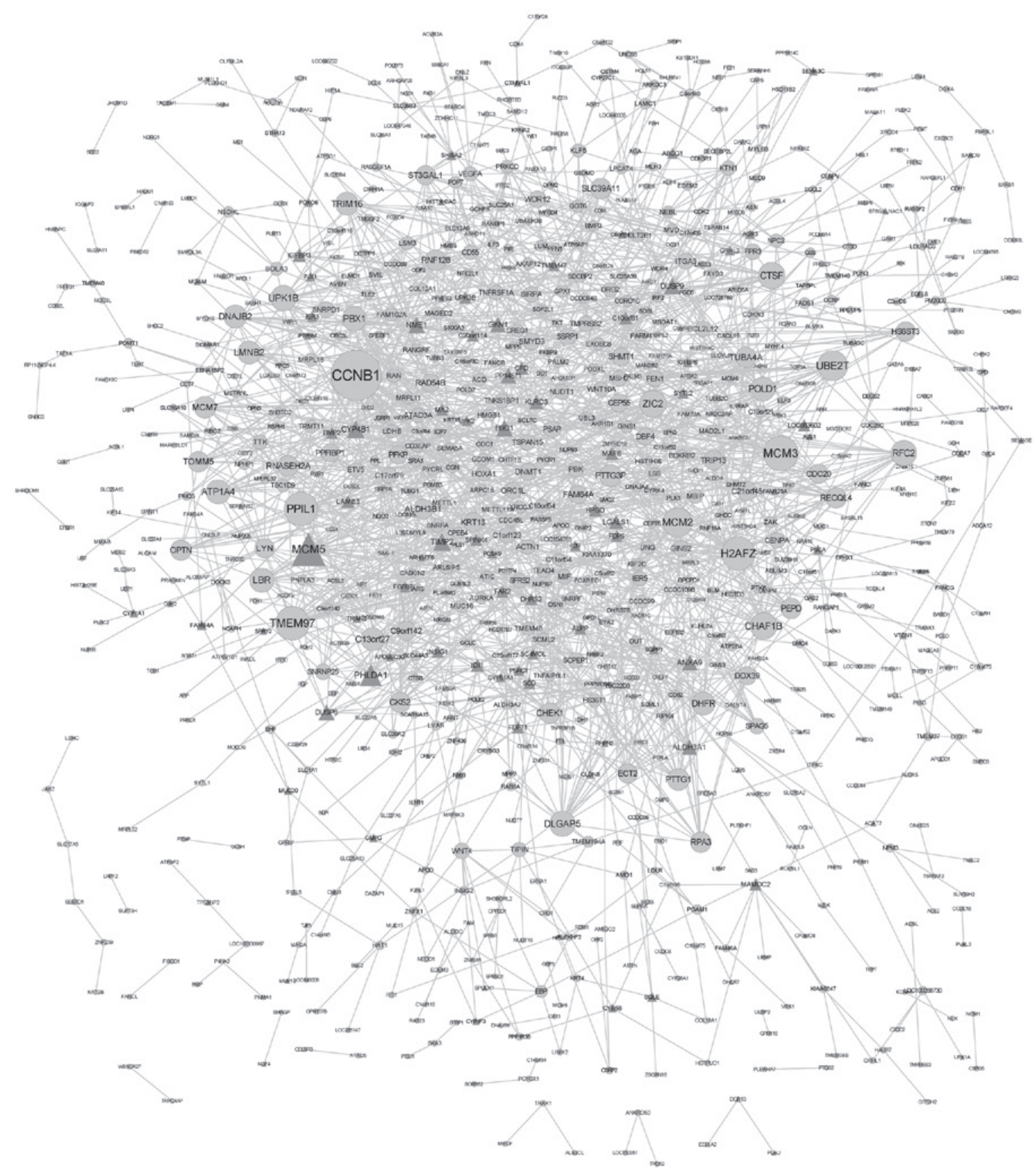

Figure 2. Gene co-expression network. A total of 938 gene nodes and 1,935 associations were identified. Triangular nodes, 140 differentially co-expressed genes; circular nodes, 798 non-differentially co-expressed genes. For each node, the degree represents the number of interactions with other nodes and node size is proportional to the degree of each node.

using Cytoscape. The network involved 1,935 co-expression associations between 140 differentially co-expressed genes and 798 non-differentially co-expressed genes (Fig. 2). Table IV lists the differentially co-expressed genes with the ten highest degrees of gene interactions, among which CCNB1 was the top hub gene with the highest degree (degree, 39).

\section{Discussion}

$\mathrm{BC}$ is the fourth most common type of solid cancer in men and the seventh most common in women, worldwide. Although mutations and overexpression of FGFR3 have been associated with $\mathrm{BC}$, the biological mechanisms underlying its pathogenesis remain to be fully elucidated. The present study aimed to analyze DEGs based on the transcription profile data of experimental FGFR3 knockdown samples and control EGFP shRNA samples. A total of 196 genes were identified to be differentially expressed in FGFR3 knockdown bladder cancer cell lines in comparison with control cell lines. GO and pathway enrichment analysis were conducted; in addition, a PPI network of DEGs and a differential co-expression network were constructed.

In the present study, the results of the GO analysis as well as the KEGG and BIOCATRA pathway enrichment analysis revealed that the primary biological process in which these DEGs were involved was sterol biosynthesis and metabolism. 
These results were consistent with those of a previous study, which demonstrated that FGFR3 may affect BC through the biosynthesis of sterol and lipids (21). Activation of FGFR3 was reported to promote the accumulation of mature sterol-regulatory element binding protein (SREBP)-1 through the PI3K-mTOR complex 1 pathway (21). SREBPs belong to the basic helix-loop-helix-leucine zipper family of transcription factors, which include SREBP-1a, SREBP-1C and SREBP-2. These transcription factors have been reported to be regulators of the activation or expression of enzymes in lipid and cholesterol homeostasis (33). Such enzymes include lanosterol-14 $\alpha$-demethylase, squalene epoxidase monooxygenase, sterol- $\Delta^{7}$-reductase, farnesyl-diphosphate farnesyl transferase 1 and sterol-C4-methyl oxidase, encoded for by CYP51A1, SQLE, DHCR7, FDFT1 and SC4MOL, respectively, which were genes identified in the present study to be involved in the top biological process affected by FGFR3 knockdown in BC, steroid biosynthesis. When sterol concentrations are low, SREBPs bind with SREBP cleavage-activating protein and mature SREBPs activate the biosynthesis of sterol (34). Wu et al (35) and Degener et al (36) revealed an association between the metabolism of steroid or bile acid and bladder cancer. Therefore, it was hypothesized that FGFR3 may regulate the corresponding target genes, which in turn affects the metabolism and biosynthesis of steroid substances and subsequently affects BC.

The genes with the ten highest degrees of interaction in the PPI network constructed in the present study were SETX, CFD, HIST2H2BE, NME2, SERPINB2, UBC, CKS, DHRS2, TRIP6 and MCM5. NME2, also known as NM23-H2, encodes nucleoside diphosphate kinase (NDPK)-B, which catalyzes the transposition of $\gamma$-phosphate between nucleosides (37). In addition, NME2 is known to be a motility and metastasis suppressor (38), which may inhibit cancer, as cells must survive and proliferate to become overt metastases. NME1, also known as NM23-H2, is a paralog of NME2, with $88 \%$ amino acid identity, and was first discovered to be a metastasis suppressor by Steeg et al (39) in 1988. Yong et al (40) studied the differential expression of NM23-H1 in BC and normal bladder cases using the immunohistochemical technique streptavidin-peroxidase procedure and demonstrated that the positive expression rates of NM23-H1 were 62.3 and $100.0 \%$ in $\mathrm{BC}$ and normal bladder, respectively. With disease progression, the positive expression rate decreased indicating its important role in BC (40). NM23 phosphorylates kinase suppressor of Ras and prevents downstream activation of the MAPK pathway (41). Therefore, FGFR3 expression may lead to the activation of the MAPK pathway. Furthermore, numerous studies have provided evidence to suggest that the NME2 gene may be associated with cancer $(42,43)$.

Co-expression genes were hypothesized to have functional associations, such as physical interactions, between the encoded proteins. In the present study, the gene co-expression network containing 168 differentially co-expressed genes and 1,935 associations were built. The genes with the highest degrees of interaction were CCNB1, MCM3, TMEM97, MCM5, H2AFZ, PPIL1, UBE2T, MCM2, CTSF and ZIC2. $\mathrm{CCNB} 1$, encoding cyclin $\mathrm{B} 1$, was reported to contribute to the regulation of $\mathrm{G}_{2}-\mathrm{M}$-phase transition, which is essential for DNA synthesis and cell proliferation (44). Dysregulated expression of CCNB1 may therefore result in uncontrolled growth and malignant transformation (45). Of note, CCNB1 is one of the 11 genes to predict outcome in several types of cancer, including BC (46). Yuan et al (47) reported that the specific downregulation of CCNB1 may lead to tumor regression through preventing the progression of cells in $\mathrm{G}_{2}$ phase and triggering cell death. H2AFZ encodes $\mathrm{H} 2 \mathrm{~A}$ histone family, member $\mathrm{Z}$, which is a variant of histone $\mathrm{H} 2 \mathrm{~A}$ and is responsible for the thermosensory response and regulating euchromatin-heterochromatin transition (48). H2AFZ may have a role in high-grade cancer due to its ability to regulate a large numbers of genes (49). Dong et al (50) revealed that $\mathrm{H} 2 \mathrm{AFZ}$ was overexpressed in $\mathrm{BC}$ and may be applied to the diagnosis of BC.

In conclusion, according to the expression profile data of FGFR3 knockdown in BC, the present study identified 196 DEGs. GO analysis as well as KEGG and BIOCATRA pathway enrichment analysis revealed that the primary biological process in which these DEGs were involved was sterol biosynthesis and metabolism. In addition, PPI networks of DEGs and co-expressed genes were constructed and revealed the information flow of PPIs. This comprehensive expression profile data of $\mathrm{BC}$ provided novel insight into the pathogenesis and occurrence of BC associated with FGFR3.

\section{References}

1. Bryan RT: Update on bladder cancer diagnosis and management. Trends in Urology and Men's Health 4: 7-11, 2013.

2. Ploeg M, Aben KK and Kiemeney LA: The present and future burden of urinary bladder cancer in the world. World J Urol 27: 289-293, 2009.

3. Billerey C, Chopin D, Aubriot-Lorton MH, et al: Frequent FGFR3 mutations in papillary non-invasive bladder ( $\mathrm{pTa}$ ) tumors. Am J Pathol 158: 1955-1959, 2001.

4. Heney NM: Natural history of superficial bladder cancer. Prognostic features and long-term disease course. Urol Clin North Am 19: 429-433, 1992.

5. Parkin DM: The global burden of urinary bladder cancer. Scand J Urol Nephrol Suppl 218: 12-20, 2008.

6. Boffetta P: Tobacco smoking and risk of bladder cancer. Scand J Urol Nephrol Suppl 218: 45-54, 2008.

7. Zeegers MP, Tan FE, Dorant E and van Den Brandt PA: The impact of characteristics of cigarette smoking on urinary tract cancer risk: A meta-analysis of epidemiologic studies. Cancer 89: 630-639, 2000.

8. Sánchez-Carbayo M and Cordon-Cardó C: Molecular alterations associated with bladder cancer progression. Semin Oncol 34: 75-84, 2007.

9. Cordon-Cardo C: Molecular alterations associated with bladder cancer initiation and progression. Scand J Urol Nephrol Suppl 218: 154-165, 2008.

10. Ornitz DM, Xu J, Colvin JS, et al: Receptor specificity of the fibroblast growth factor family. J Biol Chem 271: 15292-15297, 1996.

11. Thompson LM, Plummer S, Schalling M, et al: A gene encoding a fibroblast growth factor receptor isolated from the Huntington disease gene region of human chromosome 4 . Genomics 11: 1133-1142, 1991.

12. Perez-Castro AV, Wilson J and Altherr MR: Genomic organization of the human fibroblast growth factor receptor 3 (FGFR3) gene and comparative sequence analysis with the mouse Fgfr3 Gene. Genomics 41: 10-16, 1997.

13. Pandith AA, Shah ZA and Siddiqi MA: Oncogenic role of fibroblast growth factor receptor 3 in tumorigenesis of urinary bladder cancer. Urol Oncol 31: 398-406, 2013.

14. Turner $\mathrm{N}$ and Grose R: Fibroblast growth factor signalling: From development to cancer. Nat Rev Cancer 10: 116-129, 2010.

15. Passos-Bueno MR, Wilcox WR, Jabs EW, Sertié AL, Alonso LG and Kitoh $\mathrm{H}$ : Clinical spectrum of fibroblast growth factor receptor mutations. Hum Mutat 14: 115-125, 1999. 
16. Hernández S, de Muga S, Agell L, et al: FGFR3 mutations in prostate cancer: association with low-grade tumors. Mod Pathol 22: 848-856, 2009.

17. Rosty C, Aubriot MH, Cappellen D, et al: Clinical and biological characteristics of cervical neoplasias with FGFR3 mutation. Mol Cancer 4: 15, 2005.

18. Cappellen D, De Oliveira C, Ricol D, et al: Frequent activating mutations of FGFR3 in human bladder and cervix carcinomas. Nat Genet 23: 18-20, 1999.

19. Billerey C, Chopin D, Aubriot-Lorton MH, et al: Frequent FGFR3 mutations in papillary non-invasive bladder (pTa) tumors. Am J Pathol 158: 1955-1959, 2001

20. Jebar AH, Hurst CD, Tomlinson DC, Johnston C, Taylor CF and Knowles MA: FGFR3 and Ras gene mutations are mutually exclusive genetic events in urothelial cell carcinoma. Oncogene 24: 5218-5225, 2005.

21. Du X, Wang QR, Chan E, et al: FGFR3 stimulates stearoyl CoA desaturase 1 activity to promote bladder tumor growth. Cancer Res 72: 5843-5855, 2012.

22. Tomlinson DC, Hurst CD and Knowles MA: Knockdown by shRNA identifies S249C mutant FGFR3 as a potential therapeutic target in bladder cancer. Oncogene 26: 5889-5899, 2007.

23. Miyake M, Ishii M, Koyama N, et al: 1-tert-butyl-3-[6-(3, 5-dimethoxy-phenyl)-2-(4-diethylamino-butylamino)-pyrido [2, 3-d] pyrimidin-7-yl]-urea (PD173074), a selective tyrosine kinase inhibitor of fibroblast growth factor receptor-3 (FGFR3), inhibits cell proliferation of bladder cancer carrying the FGFR3 gene mutation along with up-regulation of p27 Kip1 and G1 G0 arrest. J Pharmacol Exp Ther 332: 795-802, 2010.

24. Irizarry RA, Hobbs B, Collin F, et al: Exploration, normalization and summaries of high density oligonucleotide array probe level data. Biostatistics 4: 249-264, 2003.

25. Smyth GK: Limma: linear models for microarray data. In Bioinformatics and Computational Biology Solutions Using R and Bioconductor. Gentleman R, Carey V, Huber W, Irizarry RA and Dudoit S (eds). Springer, New York, NY, pp397-420, 2005.

26. Benjamini $\mathrm{Y}$ and Hochberg $\mathrm{Y}$ : Controlling the false discovery rate: A practical and powerful approach to multiple testing. J Roy Statist Soc Ser B (Methodological) 57: 289-300, 1995.

27. Huang da W, Sherman BT and Lempicki RA: Systematic and integrative analysis of large gene lists using DAVID bioinformatics resources. Nat Protoc 4: 44-57, 2009.

28. Cowley MJ, Pinese M, Kassahn KS, et al: PINA v2. 0: Mining interactome modules. Nucleic Acids Res 40 (Database Issue): D862-D865, 2012

29. Shannon P, Markiel A, Ozier O, et al: Cytoscape: A software environment for integrated models of biomolecular interaction networks. Genome Res 13: 2498-2504, 2003.

30. Jansen R, Greenbaum D and Gerstein M: Relating whole-genome expression data with protein-protein interactions. Genome Res 12: 37-46, 2002

31. Kemmeren P, van Berkum NL, Vilo J, et al: Protein interaction verification and functional annotation by integrated analysis of genome-scale data. Mol Cell 9: 1133-1143, 2002.

32. Liu BH, Yu H, Tu K, Li C, Li YX and Li YY: DCGL: An R package for identifying differentially coexpressed genes and links from gene expression microarray data. Bioinformatics 26: $2637-2638,2010$
33. Yokoyama C, Wang X, Briggs MR, et al: SREBP-1, a basic-helix-loop-helix-leucine zipper protein that controls transcription of the low density lipoprotein receptor gene. Cell 75: 187-197, 1993

34. Porstmann T, Griffiths B, Chung YL, et al: PKB Akt induces transcription of enzymes involved in cholesterol and fatty acid biosynthesis via activation of SREBP. Oncogene 24: 6465-6481, 2005

35. Wu J, Liu J, Jia R and Song H: Nur77 inhibits androgen-induced bladder cancer growth. Cancer Invest 31: 654-660, 2013.

36. Degener S, Roth S, Mathers MJ and Ubrig B: Follow-up care-consequences of urinary diversion after bladder cancer. Urologe A 53: 253-262, 2014 (In German).

37. Lascu I: The nucleoside diphosphate kinases 1973-2000. J Bioenerg Biomembr 32: 211-214, 2000.

38. Rayner K, Chen YX, Hibbert B, et al: Discovery of NM23-H2 as an estrogen receptor $\beta$-associated protein: Role in estrogen-induced gene transcription and cell migration. J Steroid Biochem Mol Biol 108: 72-81, 2008.

39. Steeg PS, Bevilacqua G, Kopper L, et al: Evidence for a novel gene associated with low tumor metastatic potential. J Natl Cancer Inst 80: 200-204, 1988.

40. Yong G: Expression of C-erbB-2, EGFR and nm23-H1 in human bladder cancer and its clinical significance. Proc Clin Med 2011(5): 333-365, 2011.

41. Cook LM, Hurst DR and Welch DR: Metastasis suppressors and the tumor microenvironment. Semin Cancer Biol 21: 113-122, 2011.

42. Legin AA, Jakupec MA, Bokach NA, Tyan MR, Kukushkin VY and Keppler BK: Guanidine platinum (II) complexes: Synthesis, in vitro antitumor activity and DNA interactions. J Inorg Biochem 133: 33-39, 2014

43. Chen S, Su L, Qiu J, et al: Mechanistic studies for the role of cellular nucleic-acid-binding protein (CNBP) in regulation of c-myc transcription. Biochim Biophys Acta 1830: 4769-4777, 2013

44. Poli A, Ramazzotti G, Matteucci A, et al: A novel DAG-dependent mechanism links PKC $\alpha$ and Cyclin B1 regulating cell cycle progression. Oncotarget 30: 11526-11540, 2014

45. Soria JC, Jang SJ, Khuri FR, et al: Overexpression of cyclin B1 in early-stage non-small cell lung cancer and its clinical implication. Cancer Res 60: 4000-4004, 2000.

46. Nakamura N, Yamamoto H, Yao T, et al: Prognostic significance of expressions of cell-cycle regulatory proteins in gastrointestinal stromal tumor and the relevance of the risk grade. Hum Pathol 36: 828-837, 2005.

47. Yuan J, Krämer A, Matthess Y, et al: Stable gene silencing of cyclin B1 in tumor cells increases susceptibility to taxol and leads to growth arrest in vivo. Oncogene 25: 1753-1762, 2006.

48. Xu Y, Ayrapetov MK, Xu C, et al: Histone H2A.Z controls a critical chromatin remodeling step required for DNA double-strand break repair. Mol Cell 48: 723-733, 2012.

49. Meneghini MD, Wu M and Madhani HD: Conserved histone variant H2A.Z protects euchromatin from the ectopic spread of silent heterochromatin. Cell 112: 725-736, 2003.

50. Dong L, Bard AJ, Richards WG, Nitz MD, Theodorescu D, Bueno R and Gordon GJ: A gene expression ratio-based diagnostic test for bladder cancer. Adv Appl Bioinform Chem 2: 17-22, 2009. 